\title{
VARIABLE INTEGRAL AND SMOOTH EXPONENT TRIEBEL-LIZORKIN SPACES ASSOCIATED WITH A NON-NEGATIVE SELF-ADJOINT OPERATOR
}

\author{
JINGSHI XU AND XIAODI YANG
}

Abstract. In this paper, variable integral and smooth exponent Triebel-Lizorkin spaces associated with a non-negative self-adjoint operator are introduced. Then equivalent norms and atomic decomposition of these new spaces are given.

Mathematics subject classification (2010): 46E35, 42B25.

Keywords and phrases: Triebel-Lizorkin space, variable exponent, non-negative self-adjoint operators, maximal function, atomic decomposition.

\section{REFERENCES}

[1] T. AdAmowicz, P. HARJulehto AND P. HÄstö, Maximal operator in variable exponent Lebesgue spaces on unbounded quasimetric measure spaces, Math. Scand. 116 (2015), 5-22.

[2] A. AlmeIdA AND P. HÄstÖ, Besov spaces with variable smoothness and integrability, J. Funct. Anal. 258 (5) (2010), 1628-1655.

[3] A. Bui, J. CAO, L. D. KY, D. YANG And S. YANG, Musielak-Orlicz-Hardy spaces associated with operators satisfying reinforced off-diagonal estimates, Anal. Geom. Metric Spaces 1 (2014), 69-129.

[4] J. CAO, D. C. ChAng, H. WU AND D. YANG, Weak Hardy spaces $W H_{L}^{p}\left(\mathbb{R}^{n}\right)$ associated to operators satisfying $k$-Davies-Gaffney estimates, J. Nonlinear Convex Anal. 16 (7) (2015), 1205-1255.

[5] J. CAO, D. C. Chang, D. YAng AND S. YAnG, Boundedness of second order Riesz transforms associated to Schrödinger operators on Musielak-Orlicz-Hardy spaces, Commun. Pure Appl. Anal. 13 (2014), 1435-1463.

[6] J. CAO, D. YANG AND S. YANG, Endpoint boundedness of Riesz transforms on Hardy spaces associated with operators, Rev. Mat. Complut. 26 (2013), 99-114.

[7] Y. CHEN, S. LEVINE AND R. RAO, Variable exponent, linear growth functionals in image restoration, SIAM J. Appl. Math., 66 (4) (2006), 1383-1406.

[8] M. Christ, A T $(b)$ theorem with remarks on analytic capacity and the Cauchy integral, Colloq. Math., 60/61 (1990), 601-628.

[9] D. CruZ-Uribe and A. FiorenZa, Variable Lebesgue Spaces, Springer, Heidelberg, 2013.

[10] D. Cruz-Uribe, A. Fiorenza, J. Martell and C. PÉrez, The boundedness of classical operators on variable $L^{p}$ spaces, Ann. Acad. Sci. Fen. Math. 31 (2006), 239-264.

[11] D. Cruz-Uribe, A. Fiorenza, M. RuZhansky And J. Wirth, Variable Lebesgue spaces and Hyperbolic systems, Birkhäuser, Basel, 2014.

[12] D. CruZ-Uribe AND L. A. WANG, Variable Hardy spaces, Indiana Univ. Math. J. 63 (2) (2014), 447-493.

[13] S. Dekel, G. Kerkyacharian, G. Kyriazis and P. Petrushev, Hardy spaces associated with non-negative self-adjoint operators, arXiv:1409.0424.

[14] S. Dekel, G. Kerkyacharian, G. Kyriazis and P. Petrushev, A new proof of the atomic decomposition of Hardy spaces, arXiv:1409.0419.

[15] L. Diening, P. Harjulehto, P. HÄstö, Y. Mizuta And T. Shimomura, Maximal functions in variable exponent spaces: limiting cases of the exponent, Ann. Acad. Sci. Fenn. Math. 34 (2) (2009), 503-522. 
[16] L. Diening, P. Harjulehto, P. Hästö And M. RŮŽIČKA, Lebesgue and Sobolev spaces with variable exponents, Springer, Berlin, 2011.

[17] L. Diening, P. HÄSTÖ AND S. RoudenKo, Function spaces of variable smoothness and integrability, J. Funct. Anal. 256 (6) (2009), 1731-1768.

[18] L. Diening, Maximal function on generalized Lebesgue spaeces $L^{p^{(\cdot)}}$, Math. Inequal. Appl. 7 (2) (2004), 245-254.

[19] G. HU, Besov and Triebel-Lizorkin spaces associated with non-negative self-adjoint operators, J. Math. Anal. Appl. 411 (2014), 753-772.

[20] M. IZUKI, E. NAKAI AND Y. SAWANO, Function spaces with variable exponents an introduction, Sci. Math. Jpn. 77 (2) (2014), 187-315.

[21] R. JiAng AND D. YANG, Orlicz-Hardy spaces associated with operators satisfying Davies-Gaffney estimates, Commun. Contemp. Math. 13 (2011), 331-373.

[22] R. JiAng AND D. YANG, New Orlicz-Hardy spaces associated with divergence form elliptic operators, J. Funct. Anal. 258 (2010), 1167-1224.

[23] R. Jiang, D. YAng AND Y. Zhou, Orlicz-Hardy spaces associated with operators, Sci. China Ser. A, 52 (2009), 1042-1080.

[24] H. KempKA, 2-Microlocal Besov and Triebel-Lizorkin spaces of variable integrability, Rev. Mat. Complut. 22 (1) (2009), 227-251.

[25] H. KEMPKA, Atomic, molecular and wavelet decomposition of generalized 2-microlocal Besov spaces, J. Funct. Spaces Appl. 8 (2) (2010), 129-165.

[26] H. KempKa And J. VybíRAL, Spaces of variable smoothness and integrability: Characterizations by local means and ball means of differences, J. Fourier Anal. Appl. 18 (4) (2012), 852-891.

[27] H. KempKa AND J. VybíRAL, Lorentz spaces with variable exponents, Math. Nachr. 287 (2014), 938-954.

[28] G. Kerkyacharian And P. Petrushev, Heat kernel based decomposition of spaces of distributions in the framework of Dirichlet spaces, Trans. Amer. Math. Soc. 367 (2015), 121-189.

[29] S. G. Krantz And H. G. Parks, A Primer of Real Analytic Functions, Birkhäuser Verlag, Basel, 1992.

[30] O. Kovacik And J. Rakosnik, On spaces $L^{p(x)}$ and $W^{k, p(x)}$, Czech. Math. J. 41 (1991), 592-618.

[31] Y. Liang, D. YANG AND S. YANG, Applications of Orlicz-Hardy spaces associated with operators satisfying Poisson estimates, Sci. China Math. 54 (2011), 2395-2426.

[32] E. NAKAI AND Y. SAWANo, Hardy spaces with variable exponents and generalized Campanato spaces, J. Funct. Anal. 262 (9) (2012), 3665-3748.

[33] A. NeKVINDA, Hardy-Littlewood maximal operator on $L^{p(x)}\left(\mathbb{R}^{n}\right)$, Math. Inequal. Appl. 7 (2004), $255-265$.

[34] M. RUŽIČKA, Electrorheological Fluids: Modeling and Mathematical Theory, Springer-Verlag, Berlin, 2000.

[35] V. S. Rychkov, On a theorem of Bui, Paluszyński, and Taibleson, Proc. Steklov Inst. Math. 227 (1999), 280-292.

[36] H. TRIEBEL, Theory of function spaces, Birkhäuser, Basel, 1983.

[37] H. TRIEBEL, Theory of function spaces II, Birkhäuser, Basel, 1992.

[38] H. TRIEBEL, The structure of functions, Birkhäuser, Basel, 2001.

[39] H. TRIEBEL, Theory of function spaces III, Birkhäuser, Basel, 2006.

[40] T. UlLRICH, Continuous characterizations of Besov-Lizorkin-Triebel spaces and new interpretations as coorbits, J. Funct. Spaces Appl. 2012 (2012), Article ID 163213, 47 pages.

[41] J. Xu, Variable Besov spaces and Triebel-Lizorkin spaces, Ann. Acad. Sci. Fenn. Math. 33 (2) (2008), $511-522$.

[42] D. YAng And Do. YAng, Maximal function characterizations of Musielak-Orlicz-Hardy spaces associated with magnetic schrödinger operators, Front. Math. China. 10 (5) (2015), 1203-1232.

[43] D. YANG AND S. YANG, Orlicz-Hardy spaces associated with divergence operators on unbounded strongly Lipschitz. domains of $\mathbb{R}^{n}$, Indiana Univ. Math. J. 61 (2012), 81-129.

[44] D. YANG AND S. YANG, Real-variable characterizations of Orlicz-Hardy spaces on strongly Lipschitz domains of $\mathbb{R}^{n}$, Rev. Mat. Iberoam. 29 (2013), 237-292.

[45] D. Yang And S. Yang, Local Hardy spaces of Musielak-Orlicz type and their applications, Sci. China Math. 55 (2012), 1677-1720.

[46] D. YANG AND S. YANG, Musielak-Orlicz-Hardy spaces associated with operators and their applications, J. Geom. Anal. 24 (2014), 495-570. 
[47] D. YANG AND S. YANG, Second-order Riesz transforms and maximal inequalities associated to magnetic Schrödinger operators, Canad. Math. Bull. 58 (2015), 432-448.

[48] J. ZhANG, J. CAO, R. JIANG AND D. YANG, Non-tangential maximal function characterizations of Hardy spaces associated to degenerate elliptic operators, Canad. J. Math. 67 (2015), 1161-1200. 